\title{
Vedolizumab como terapia primaria en colitis ulcerosa y en la enfermedad de Crohn de moderada a grave: revisión y metaanálisis
}

\section{Vedolizumab as primary therapy in ulcerative colitis and moderate to severe Crohn's disease: review and meta-analysis}

\author{
Rebeca Pérez-Cabeza de Vaca ${ }^{1 *}$, Tomás Cortés-Espinoza², Brenda Maldonado-Arriaga ${ }^{3}$, \\ Juan A. Suárez-Cuenca ${ }^{3}$, Sergio Sandoval-Jiménez ${ }^{4}$, Paul Mondragón-Terán ${ }^{5}$, Christian Navarro-Gerrard², \\ Carlos Paredes-Amenábar², Korely Corazón-Trujillo de la Fuente², José A. Romero-Lozanía², \\ Jesús G. López-Gómez² y Cuauhtémoc Licona-Cassanib
}

${ }^{1}$ División de Investigación Biomédica, Centro Médico Nacional 20 de Noviembre, Instituto de Seguridad y Servicios Sociales de los Trabajadores del Estado, Ciudad de México; 2 Servicio de Gastroenterología, Centro de Enfermedad Inflamatoria Intestinal, Centro Médico Nacional 20 de Noviembre, Instituto de Seguridad y Servicios Sociales de los Trabajadores del Estado, Ciudad de México; ${ }^{3}$ Laboratorio de Metabolismo Experimental e Investigación Clínica, Centro Médico Nacional 20 de Noviembre, Instituto de Seguridad y Servicios Sociales de los Trabajadores del Estado, Ciudad de México; " ${ }^{S}$ Servicio de Endoscopía Gastrointestinal, Hospital General de 2A Troncoso, Instituto Mexicano del Seguro Social, Ciudad de México; ${ }^{5}$ Servicio de Coordinación de Investigación, Centro Médico Nacional 20 de Noviembre, Instituto de Seguridad y Servicios Sociales de los Trabajadores del Estado, Ciudad de México; ${ }^{6}$ Laboratorio de Genómica Industrial, Centro de Biotecnología FEMSA, Tecnológico de Monterrey, Monterrey, Nuevo León. México

\section{Resumen}

Antecedentes: La colitis ulcerosa (CU) y la enfermedad de Crohn (EC) tienen diferentes opciones de tratamiento, que incluyen aminosalicilatos, corticosteroides, tiopurinas, inhibidores de calcineurina y biológicos como anticitocinas y antiintegrinas. Y recientemente el uso de vedolizumab (VDZ), el cual es un anticuerpo monoclonal humanizado selectivo para el intestino que se une a las integrinas $\alpha 4 \beta 7$, bloqueando selectivamente el tráfico de linfocitos en el intestino. El objetivo de este metaanálisis fue estimar la eficacia y seguridad del fármaco VDZ en ensayos clínicos aleatorizados en pacientes adultos no tratados previamente con terapia biológica y corticosteroides con CU y EC. Metodología: Se realizó una revisión sistemática de la literatura y un metaanálisis para evaluar la efectividad y seguridad del VDZ en pacientes con CU y EC. Se realizó una búsqueda en las bases de datos PubMed/Medline sobre pacientes adultos con CU sin previo uso de terapia biológica y corticosteroides bajo tratamiento de VDZ en ensayos clínicos aleatorizados. Resultados: Se seleccionaron cinco estudios que cumplieron los criterios de selección. Y se determinó que en la semana 14 de tratamiento, el $32 \%$ de los pacientes con CU estaban en remisión; y a los 12 meses, el 46\% estaba en remisión clínica. Las tasas de remisión sin corticosteroides fueron del $26 \%$ en la semana 14 y del $42 \%$ en el mes 12. En el mes 12, del 33 al 77\% de los pacientes con CU presentaron curación de la mucosa. El 9\% de los pacientes informaron eventos adversos graves. Conclusiones: EI VDZ demostró eficacia en pacientes con CU y EC activa de moderada a grave, logrando la remisión en el mes 12 de tratamiento. Estos hallazgos concuerdan con los datos y el apoyo de los ensayos clínicos aleatorizados muestran el perfil de riesgo-beneficio a largo plazo del VDZ.

Palabras clave: Metaanálisis. Vedolizumab. Colitis ulcerativa. Corticosteroides. Terapia biológica.

Correspondencia:

*Rebeca Pérez-Cabeza de Vaca

E-mail: esderebk@gmail.com
Disponible en internet: 01-09-2021

Fecha de recepción: 11-05-2021

Fecha de aceptación: 24-06-2021

DOI: 10.24875/IMIDS.M21000006
Rev Mex Enferm Inflam Inmunomed. 2021:95(2):31-39

www.IMIDsMexico.com

2696-6867 / @ 2021 Permanyer. Éste es un artículo open access bajo la licencia CC BY-NC-ND (http://creativecommons.org/licenses/by-nc-nd/4.0/) 


\section{Abstract}

Background: Ulcerative colitis (UC) and Crohn disease (CD) has different treatment options including aminosalicylates, corticosteroids (CS), thiopurines, calcineurin inhibitors, and biological as anti-cytokines, and anti-integrins. And recently the use of vedolizumab (VDZ) which is a humanized monoclonal antibody selective for the intestine that binds to the $\alpha 4 \beta 7$ integrins, selectively blocking the traffic of selective lymphocytes in the intestine. The objective of this meta-analysis was to estimate the efficacy and safety of the drug VDZ in randomized clinical trials in adult patients not previously treated with biological therapy and corticosteroids with UC and CD. Methodology: A systematic review of the literature and a meta-analysis were carried out to evaluate the effectiveness and safety of VDZ in patients with UC and CD. A search was carried out in the PubMed/Medline databases on adult patients with UC without previous use of biological therapy and corticosteroids under treatment of VDZ in randomized clinical trials. Results: Five studies were selected that met the selection criteria. And it was determined that at week 14 of treatment, $32 \%$ of UC patients were in remission; and at 12 months, 46\% were in clinical remission. Corticosteroid-free remission rates were $26 \%$ at week 14 and $42 \%$ at month 12 . At month 12,33 to $77 \%$ of UC patients had mucosal healing. Nine percent of the patients reported serious adverse events. Conclusions: VDZ demonstrated efficacy in patients with moderate to severe active UC and $C D$, achieving remission in month 12 of treatment. These findings are consistent with data and support from randomized clinical trials demonstrating the long-term benefit-risk profile of VDZ.

Key words: Meta-analysis. Vedolizumab. Ulcerative colitis. Corticosteroids. Biological therapy.

\section{Introducción}

La colitis ulcerosa (CU) y la enfermedad de Crohn (EC) se consideran enfermedades de tipo inflamatorio crónico que involucran cualquier porción del tracto gastrointestinal. Son enfermedades de etiología multifactorial, destacando al factor hereditario, el factor ambiental y la microbiota intestinal. Se caracterizan por la presencia de inflamación crónica difusa en la mucosa colónica ${ }^{1}$. El curso clínico de la CU se distingue por periodos de exacerbación y remisión, y su principal síntoma es la diarrea sanguinolenta asociada con urgencia y tenesmo rectal. Mientras que la EC causa daño estructural en el tracto gastrointestinal que resulta en complicaciones de estenosis, fístula y formación de abscesos ${ }^{2}$.

El tratamiento consiste en manipulaciones dietéticas, diferentes fármacos y en ocasiones tratamiento quirúrgico, y debe adaptarse a la severidad, segmento intestinal afectado, historia evolutiva, estado nutricional y la existencia de potenciales complicaciones ${ }^{3,4}$. Los objetivos principales de los tratamientos para estas patologías son inducir y mantener un estado de remisión clínica, y prevenir las recaídas ${ }^{5}$.

En el tratamiento farmacológico destaca el uso de aminosalicilatos como primera opción en la CU y en la EC de leve a moderada; se pueden utilizar en la etapa de inducción a la remisión, mantenimiento y recaída. Su principal mecanismo de acción es la inhibición de la vía de la lipooxigenasa en el metabolismo del ácido araquidónico por medio de la producción del leucotrieno B4 y actúa preferentemente sobre el colon y parcialmente sobre el íleon terminal6-8.

Recientemente, el uso de la terapia biológica se ha incrementado en los últimos años, debido a los nuevos hallazgos sobre los mecanismos de patogenicidad de la CU que están dirigidos directamente a mediadores proinflamatorios, como el factor de necrosis tumoral alfa (TNF- $\alpha$ ), citocinas y agentes de migración de membranas, entre otros ${ }^{9}$. Los anticuerpos monoclonales son la última opción farmacoterapéutica para los pacientes con CU y EC antes de que la cirugía sea inevitable. Entre los anticuerpos monoclonales más utilizados para la CU y la EC de moderada a grave destacan: infliximab (IFX), adalimumab (ADA), ustekinumab (UST) y vedolizumab (VDZ) de manera reciente ${ }^{10}$.

EI IFX es un anticuerpo monoclonal quimérico contra el TNF- $\alpha$, administrado por vía intravenosa a una dosis estándar de $5 \mathrm{mg} / \mathrm{kg}$ en las semanas 0, 2, 6 y luego cada 8 semanas. El tratamiento con IFX ha resultado ser eficaz en la inducción de la remisión clínica, la respuesta clínica y la curación de la mucosa en la semana 8, así mismo se ha demostrado que se puede utilizar como tratamiento de mantenimiento ${ }^{11,12}$.

EI ADA es un anticuerpo monoclonal contra TNF- $\alpha$ que se administra por vía subcutánea a una dosis de inducción estándar de 160 mg seguida de 80 mg después de 2 semanas y luego $40 \mathrm{mg}$ cada 2 semanas. Se ha descrito que es eficaz para lograr la remisión clínica sin esteroides y la curación mucosa dependiente de corticosteroides. Sin embargo, el uso previo 
de IFX es un factor predictivo de menor eficacia. Adicionalmente, el uso de ADA disminuye los niveles de la proteína $C$ reactiva y de calprotectina fecal, reflejando, de forma indirecta, el control sobre la inflamación y la curación mucosa ${ }^{13}$.

EI UST es un anticuerpo monoclonal contra la subunidad p40 de la interleucina (IL) 12 y la IL-23, se administra por vía intravenosa a una dosis de $130 \mathrm{mg}$, como terapia de inducción de 8 semanas y terapia de mantenimiento de 44 semanas en pacientes con CU y EC de moderada a grave. Se ha descrito que el uso UST logra la cicatrización histológica y endoscópica de la mucosa después de la terapia de inducción y se asocia con una menor actividad de la enfermedad al final de la terapia de mantenimiento ${ }^{14}$.

Finalmente, el VDZ es un anticuerpo monoclonal que reconoce específicamente el heterodímero de integrina $\alpha 4 \beta 7$ y bloquea selectivamente el tráfico de linfocitos intestinales sin interferir con el tráfico hacia el sistema nervioso central. Se ha demostrado su eficacia para inducir y mantener la remisión en pacientes con CU y $E C$, en los que fracasó el tratamiento previo con corticosteroides, tiopurinas 0 agentes anti-TNF ${ }^{15}$. Sin embargo, es necesario determinar el uso de VDZ como terapia primaria en la CU y EC de moderada a grave, y sí impacta de manera positiva en la recuperación de los pacientes con bajos efectos secundarios. Por lo que, en el presente estudio, el objetivo fue realizar una revisión y metaanálisis de la evidencia que existe respecto al uso del fármaco VDZ (inhibidor de la integrina $\alpha 4 \beta 7)$ como terapia primaria y su eficacia en el tratamiento de CU y EC de moderada a grave.

\section{Metodología}

El trabajo se realizó bajo las directrices del manual de Cochrane y la declaración PRISMA-NMA (Preferred Reporting Items for Systematic Reviews and MetaAnalyses for Network Meta-Analyses) para revisiones sistemáticas que incorporan redes de metaanálisis.

\section{Búsqueda y selección de estudios}

Mediante la base de datos de PubMed/Medline se realizó la búsqueda de los siguientes términos: vedolizumab, colitis ulcerosa, enfermedad de Crohn, moderada a grave, terapia primaria y ensayos clínicos aleatorizados. La búsqueda se limitó a ensayos clínicos aleatorizados realizados en seres humanos. No se consideró restricción de idioma para la revisión de los estudios y los criterios de inclusión a detalle fueron: aquellos estudios aleatorizados y controlados, con placebo, que evaluaran la eficacia o el daño de VDZ para el tratamiento de CU y la EC moderada a grave en adultos, sin previo uso de corticosteroides y anti TNF- $\alpha$, sin importar el país y la fase del ensayo clínico aleatorizado. La CU moderada a grave se definió con base en la puntuación del índice Mayo (6 a 12 puntos), el cual representa la sumatoria de la frecuencia de deposiciones, hemorragia rectal, hallazgos endoscópicos y la evaluación general del médico especialista. Por otro lado, en la EC se definió como brote moderado (Clinical Disease Activity Index [CDAl]: 220-450) la presencia de dolor a la palpación abdominal, febrícula, anemia o pérdida de peso superior a $10 \%$; y como brote grave (CDAl > 450) si el paciente requirió ser hospitalizado por caquexia, fiebre, vómitos, obstrucción intestinal, signos peritoneales o colección intraabdominal. Los criterios de exclusión fueron: comparación de VDZ con otros fármacos, pacientes con exposición previa a antagonista del TNF- $\alpha$.

\section{Extracción de datos}

Dos autores extrajeron de forma independiente la siguiente información: apellido del primer autor, ubicación geográfica del estudio y año de publicación, diseño del estudio, número de participantes, características de la población, dosis y administración del fármaco. Los dos investigadores revisaron publicaciones relevantes de forma independiente, si no estaban satisfechos con la selección de los artículos, esto se resolvió mediante una discusión o un tercer revisor.

Adicionalmente, las diferentes dosis se trataron como diferentes intervenciones, y para las terapias biológicas solo se consideraron los datos de dosis y vía de administración. Por otro lado, se evaluó la eficacia la respuesta clínica, remisión clínica, curación de la mucosa, en la fase de inducción y en la fase de mantenimiento. La respuesta clínica se definió como una disminución del índice Mayo (3 puntos y una disminución del sangrado rectal), así como una disminución en el índice de actividad de la EC. La remisión clínica se definió como una disminución del índice Mayo y del índice de actividad de la EC. La curación de la mucosa se definió con un subpuntaje de endoscopia absoluta de 0 o 1. Los desacuerdos entre los revisores se resolvieron mediante consenso.

\section{Análisis estadístico}

Todos los análisis se realizaron teniendo en cuenta el tamaño de muestra adecuado para cada resultado, 
Tabla 1. Características de los estudios que evalúan la terapia con vedolizumab (VDZ) en pacientes con colitis ulcerosa (CU) y enfermedad de Crohn (EC)

\begin{tabular}{|c|c|c|}
\hline Estudio (CU) & Resultado & Conclusión \\
\hline $\begin{array}{l}\text { Lam, et al., } 2014^{16} \\
\mathrm{~N}=885\end{array}$ & $\begin{array}{l}\text { Remisión: } 52.5 \% \\
\text { Mejoría endoscópica: } 51.6 \%\end{array}$ & $\begin{array}{l}\text { EI VDZ es eficaz para inducción y mantenimiento de CU de } \\
\text { moderada a grave en pacientes que no han recibido tratamiento } \\
\text { previo }\end{array}$ \\
\hline $\begin{array}{l}\text { Loftus, et al., } 2020^{17} \\
\mathrm{~N}=454\end{array}$ & Remisión: 10.2\% & $\begin{array}{l}\text { Pacientes con CU tratados con VDZ lograron una remisión clínica } \\
\text { sostenida }\end{array}$ \\
\hline $\begin{array}{l}\text { Motoya, et al., } 2019^{18} \\
\mathrm{~N}=292\end{array}$ & $\begin{array}{l}\text { Remisión: } 72.5 \% \\
\text { Mantenimiento: } 56.1 \% \\
\text { Efectos adversos: } 6.1 \%\end{array}$ & $\begin{array}{l}\text { El VDZ mostró eficacia como terapia de inducción y como terapia } \\
\text { de mantenimiento en pacientes japoneses con CU. Y presenta } \\
\text { seguridad y tolerabilidad favorable }\end{array}$ \\
\hline $\begin{array}{l}\text { Sandborn, et al., } 2020^{19} \\
N=216\end{array}$ & $\begin{array}{l}\text { Remisión: } 88.8 \% \\
\text { Mejoría endoscópica: } 74.2 \% \\
\text { Efectos adversos: } 12.2 \%\end{array}$ & $\begin{array}{l}\text { EI VDZ es eficaz como terapia de mantenimiento en pacientes } \\
\text { con CU activa de moderada a grave. Tiene un perfil de seguridad } \\
\text { y tolerabilidad favorable }\end{array}$ \\
\hline $\begin{array}{l}\text { Sands, et al., } 2019^{14} \\
N=383\end{array}$ & $\begin{array}{l}\text { Remisión: } 31.3 \% \\
\text { Mejoría endoscópica: } 39.7 \% \\
\text { Efectos adversos: } 23.4 \%\end{array}$ & $\begin{array}{l}\text { El uso de VDZ en la CU logró la remisión clínica y la mejoría } \\
\text { endoscópica }\end{array}$ \\
\hline Estudio (EC) & Resultado & Conclusión \\
\hline $\begin{array}{l}\text { Sands, et al., } 2017^{20} \\
N=263\end{array}$ & $\begin{array}{l}\text { Remisión (respondedores): } 48.9 \% \\
\text { Remisión (no respondedores): } 27.7 \% \\
\text { Efectos adversos: } 5.6 \%\end{array}$ & $\begin{array}{l}\text { El VDZ tuvo una mayor eficacia con EC independientemente del } \\
\text { historial de tratamiento con antagonistas del TNF- } \alpha\end{array}$ \\
\hline $\begin{array}{l}\text { Danese, et al., } 2019^{21} \\
\mathrm{~N}=191\end{array}$ & $\begin{array}{l}\text { Remisión: } 21.9 \% \\
\text { Mejoría endoscópica: } 17.9 \% \\
\text { Efectos adversos: } 8.4 \%\end{array}$ & $\begin{array}{l}\text { El tratamiento con VDZ induce la curación endoscópica, } \\
\text { radiológica e histológica en pacientes con EC activa de } \\
\text { moderada a grave }\end{array}$ \\
\hline $\begin{array}{l}\text { Watanabe, et al., } 2020^{22} \\
N=157\end{array}$ & $\begin{array}{l}\text { Remisión: } 41.7 \% \\
\text { Efectos adversos: } 10.1 \%\end{array}$ & $\begin{array}{l}\text { El VDZ mostró eficacia como terapia de inducción y terapia de } \\
\text { mantenimiento en pacientes con EC, y fue bien tolerado }\end{array}$ \\
\hline
\end{tabular}

TNF- $\alpha$ : factor de necrosis tumoral alfa.

ya que se informaron diferentes tamaños de muestra para los diversos resultados evaluados en los estudios incluidos en el metaanálisis, dado su diseño retrospectivo. La heterogeneidad entre los estudios se evaluó mediante $\mathrm{I}^{2}$ y el intervalo de confianza (IC) del 95\%. Debido al escaso número de estudios incluidos en el metaanálisis, no se realizaron análisis de metarregresión y no se evaluó el sesgo de publicación. Todos los análisis estadísticos se realizaron utilizando el paquete metafor para $\mathrm{R}$ (versión 3.4.2).

\section{Resultados}

De 48 publicaciones identificadas, se revisaron 13 publicaciones y 8 fueron seleccionadas para ser incluidas en el metaanálisis. La mayoría de los estudios fueron realizados en los EE.UU., seguido de Europa y Japón (Tabla 1). Los estudios del metaanálisis describieron una remisión clínica del $51.06 \%$, y el $55.1 \%$ informaron la curación de la mucosa o mejoría endoscópica. Seis estudios informaron sobre seguridad del VDZ, reportando un $13.9 \%$ de esta.
De la totalidad de los estudios, la edad media de los pacientes fue de 41.4 años, la duración media de la enfermedad fue de 7.5 años y el porcentaje de pacientes con tratamiento previo anti-TNF- $\alpha /$ corticosteroides fue del $21.6 \%$. Los datos demográficos de los pacientes se describen en la tabla 2.

\section{Remisión clínica}

La remisión clínica se logró en el $41 \%$ de los pacientes a la semana 6 (IC 95\%: 13-41\%) y en el $26 \%$ en la semana 14 (IC 95\%: 27-39\%), que aumentó al 48\% a los 6 meses (IC 95\%: 30-48\%) y al 59\% a los 12 meses (IC 95\%: 37-56\%) (Fig. 1). La heterogeneidad entre estudios fue evidente para todos los grupos incluidos en los análisis de remisión de CU y EC.

\section{REMISIÓN CLÍNICA SIN ANTI-TNF- $\alpha$ l CORTICOSTEROIDES}

Se evaluó la remisión clínica libre de anti-TNF- $\alpha$ / corticosteroides, se logró en el $14 \%$ en la semana 6 
Tabla 2. Datos demográficos de los pacientes con colitis ulcerosa (CU) y enfermedad de Crohn (EC) que utilizaron como terapia primaria el vedolizumab (VDZ)

\begin{tabular}{|c|c|c|c|c|}
\hline Estudio (CU) & Edad (años) & Sexo (M)\% & $\begin{array}{l}\text { Duración de la } \\
\text { enfermedad (años) }\end{array}$ & $\begin{array}{c}\text { Tratamiento previo anti-TNF- } \alpha \text { y/o } \\
\text { corticosteroides }(\%)\end{array}$ \\
\hline $\begin{array}{l}\text { Lam, et al., } 2014^{16} \\
\mathrm{~N}=885\end{array}$ & 41.0 & 45.3 & 7.1 & 21 \\
\hline $\begin{array}{l}\text { Loftus, et al., } 2020^{17} \\
N=454\end{array}$ & 40.6 & 56.8 & 6.5 & 32 \\
\hline $\begin{array}{l}\text { Motoya, et al., } 2019^{18} \\
\mathrm{~N}=292\end{array}$ & 43.0 & 51.5 & 8.6 & 23 \\
\hline $\begin{array}{l}\text { Sandborn, et al., } 2020^{19} \\
N=216\end{array}$ & 41.6 & 65.2 & 8.0 & 12 \\
\hline $\begin{array}{l}\text { Sands, et al., } 2019^{14} \\
N=383\end{array}$ & 40.8 & 60.8 & 7.3 & 20 \\
\hline Total & $41.4 \pm 2.7$ & $55.9 \pm 5.1$ & $7.5 \pm 1.2$ & $21.6 \pm 7.2$ \\
\hline Estudio (CU) & Edad (años) & $\operatorname{Sexo}(M) \%$ & $\begin{array}{l}\text { Duración de la } \\
\text { enfermedad (años) }\end{array}$ & $\begin{array}{c}\text { Tratamiento previo anti-TNF- } \alpha \text { y/o } \\
\text { corticosteroides (\%) }\end{array}$ \\
\hline $\begin{array}{l}\text { Sands, et al., } 2017^{20} \\
N=263\end{array}$ & 36.6 & 45.8 & 9.5 & 35.2 \\
\hline $\begin{array}{l}\text { Danese, et al., } 2019^{21} \\
\mathrm{~N}=191\end{array}$ & 38 & 43 & 11.4 & 75.5 \\
\hline $\begin{array}{l}\text { Watanabe, et al., } 2020^{22} \\
N=157\end{array}$ & 35.2 & 52 & 9.1 & 61 \\
\hline Total & $36.6 \pm 5.4$ & $46.9 \pm 4.3$ & $10 \pm 1.3$ & $57.2 \pm 8.2$ \\
\hline
\end{tabular}

TNF- $\alpha$ : factor de necrosis tumoral alfa.

(IC 95\%: 6-32\%), en el 26\% en semana 14 (IC 95\%: $20-34 \%$ ) y en el $32 \%$ a los 6 meses (IC 95\%: 21-45\%), con una tasa que aumenta al $42 \%$ a los 12 meses (IC 95\%: 31-53\%) (Fig. 2). La heterogeneidad entre estudios fue evidente para todos los grupos.

\section{Curación de la mucosa y mejora endoscópica}

Las tasas de curación de la mucosa variaron del 24 al $55 \%$ en pacientes con CU en el sexto mes. En el doceavo mes, las tasas de curación de la mucosa variaron del 33 al $77 \%$ en pacientes con CU y la EC (Fig. 3).

\section{Seguridad del vedolizumab}

Los resultados de seguridad se informaron durante un periodo de exposición de 1 a 12 meses. Los eventos adversos más comunes fueron artralgia, dolor de cabeza y fatiga, entre otros. Adicionalmente, dos estudios presentaron infecciones por Clostridium difficile y en menor proporción reportaron otras infecciones en el tracto respiratorio y en el tracto genitourinario (Tabla 3).

\section{Discusión}

El biológico VDZ ha demostrado ser eficaz en pacientes con CU de moderada a grave que presentan respuesta inadecuada, pérdida de respuesta o intolerancia a la terapia convencional o a la terapia anti-TNF- $\alpha$ tanto en la fase de inducción como en la de mantenimiento, por lo que en este metaanálisis se evalúa la evidencia respecto a la eficacia y seguridad del VDZ como terapia primaria en la $\mathrm{CU}$ y en la EC de moderada a grave $^{16,17}$.

En los ensayos clínicos aleatorizados seleccionados se logró la remisión clínica en aproximadamente un tercio de los pacientes a las 14 semanas y en aproximadamente la mitad de los pacientes a los 12 meses. Un objetivo importante del tratamiento en los pacientes con la enfermedad inflamatoria intestinal (EII) es lograr el mantenimiento y una remisión clínica 


\section{A}

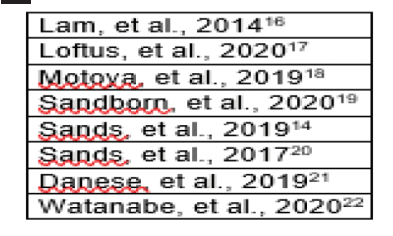

B

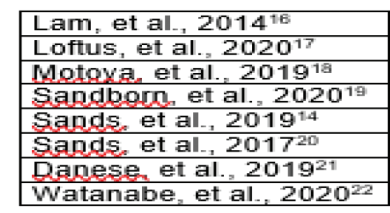



Pacientes en

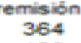

128

142

71

201

241

125

103
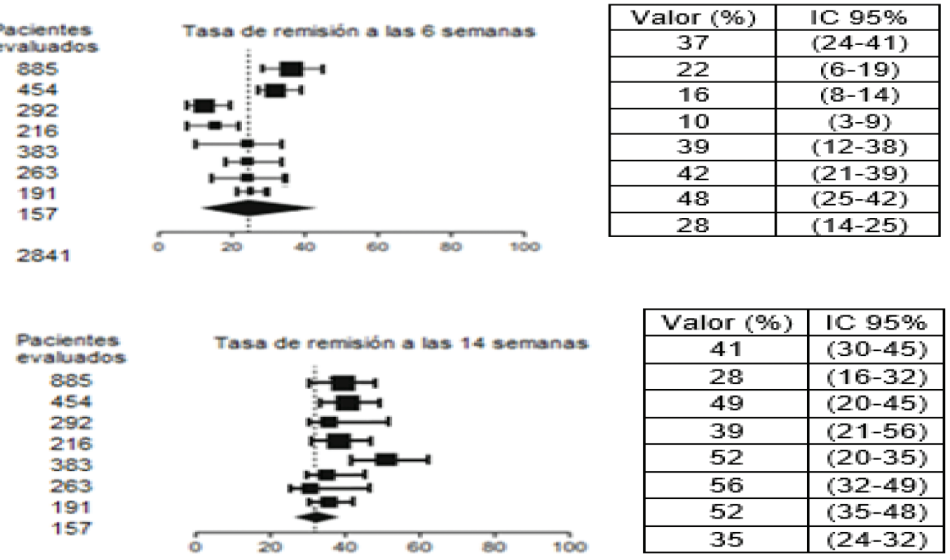

C
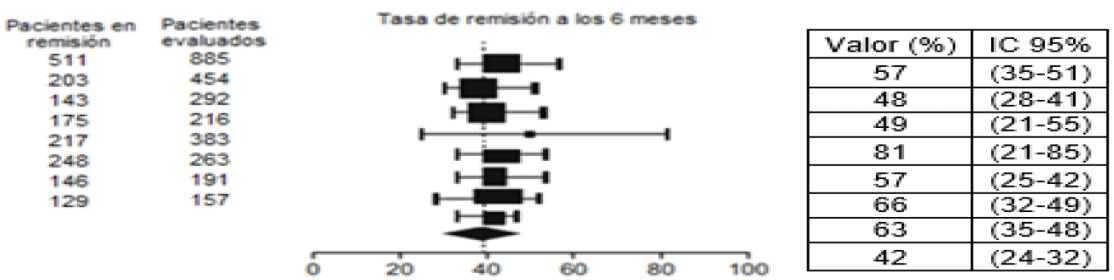

Heterogeneidad $p=0.01$

\section{D}

\begin{tabular}{|c|}
\hline Lam, et al, 20 \\
\hline Loftus, et al., $2020^{17}$ \\
\hline Motoxa et al., $2019^{1 a}$ \\
\hline Sapdbow, et al., $2020^{19}$ \\
\hline Sands, et al, $2019^{14}$ \\
\hline Sapds, et al., $2017^{20}$ \\
\hline Danese, et al., $2019^{21}$ \\
\hline Watanabe, et al., 2020 \\
\hline
\end{tabular}

Heterogeneidad $p<0.01$
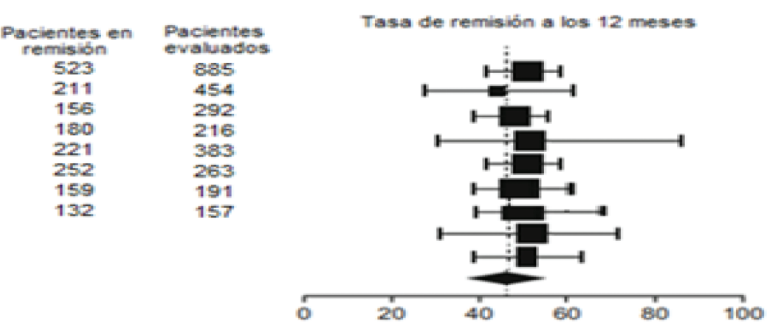

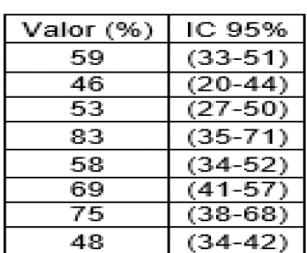

$48 \quad(34-42)$

Figura 1. Metaanálisis de las tasas de remisión clínica entre pacientes con colitis ulcerosa y pacientes con enfermedad de Crohn que recibieron vedolizumab en diferentes tiempos. A: remisión semana 6. B: remisión semana 14. C: remisión 6 meses. D: remisión 12 meses. El tamaño de cada cuadrado representa el peso asignado a cada estudio según el tamaño de la muestra. Las barras de error representan el intervalo de confianza (IC) del 95\%. Los diamantes representan la estimación puntual de las tasas de estudio promediadas; las puntas laterales de los diamantes representan el IC del 95\%.

sin el uso de corticosteroides ${ }^{18-20}$. Aproximadamente el $42 \%$ de los pacientes con CU y EC lograron una remisión clínica sin corticosteroides a la semana 14. Por tanto, la remisión clínica en la semana 14 puede ser un factor de predicción de la remisión clínica sostenida con VDZ. Es importante mencionar que los pacientes que componen la cohorte presentaban una fase grave y refractaria al tratamiento, y puntualizar que la mayoría no había tenido éxito con el tratamiento anti-TNF- $\alpha$. Con base en los ensayos clínicos aleatorizados, se debe lograr una mayor efectividad en pacientes sin experiencia biológica. En un entorno del mundo real, esta tendencia podría inducir tasas de eficacia más altas con el uso continuo y temprano del fármaco ${ }^{21,22}$. Además, en nuestro estudio, hasta aproximadamente un tercio de los pacientes con CU y EC lograron la remisión clínica después de la semana 14, lo que sugiere posibles beneficios de la monitorización terapéutica más allá de este punto de tiempo 23,24 .

Estos resultados refuerzan aún más la evidencia de que el VDZ muestra una mayor eficacia en pacientes 


\section{A}

Estudio

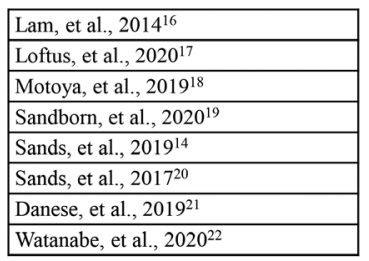

Heterogeneidad $\mathrm{p}<0.0001$

B

Estudio

\begin{tabular}{|l|}
\hline Lam, et al., 2014 \\
\hline Loftus, et al., $2020^{17}$ \\
\hline Motoya, et al., $2019^{18}$ \\
\hline Sandborn, et al., $2020^{19}$ \\
\hline Sands, et al., 201914 \\
\hline Sands, et al., 201720 \\
\hline Danese, et al., 201921 \\
\hline Watanabe, et al., $2020^{22}$ \\
\hline
\end{tabular}

Heterogeneidad $\mathrm{p}<0.0001$
Tasa de remisión a la semana 14

$\begin{array}{cc}\begin{array}{c}\text { Pacientes en } \\ \text { remision }\end{array} & \begin{array}{c}\text { Pacientes } \\ \text { evaluados }\end{array} \\ 85 & 125 \\ 61 & 136 \\ 15 & 56 \\ 18 & 48 \\ 42 & 96 \\ 41 & 86 \\ 36 & 58 \\ 42 & 74\end{array}$
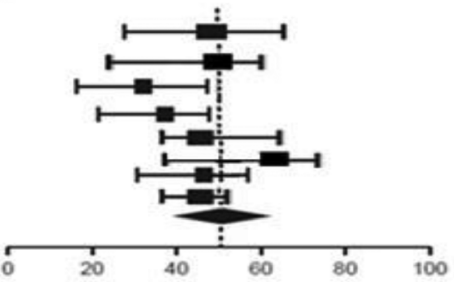

Tasa de remisión a los 12 meses.

$\begin{array}{cc}\begin{array}{c}\text { Pacientes en } \\ \text { remisión }\end{array} & \begin{array}{c}\text { Pacientes } \\ \text { ovaluados }\end{array} \\ 101 & 151 \\ 85 & 167 \\ 28 & 89 \\ 34 & 91 \\ 72 & 114 \\ 58 & 161 \\ 67 & 189 \\ 71 & 159\end{array}$

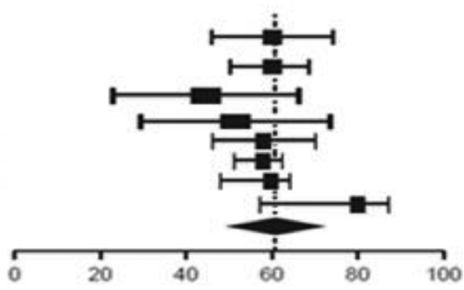

\begin{tabular}{|c|c|}
\hline Valor (\%) & IC 95\% \\
\hline 68 & $(21-54)$ \\
\hline 45 & $(20-60)$ \\
\hline 28 & $(19-32)$ \\
\hline 38 & $(20-32)$ \\
\hline 44 & $(40-59)$ \\
\hline 31 & $(28-38)$ \\
\hline 42 & $(37-48)$ \\
\hline 40 & $(32-51)$ \\
\hline
\end{tabular}

\begin{tabular}{|c|c|}
\hline Valor (\%) & IC 95\% \\
\hline 67 & $(45-72)$ \\
\hline 51 & $(42-60)$ \\
\hline 31 & $(20-42)$ \\
\hline 37 & $(35-71)$ \\
\hline 63 & $(52-70)$ \\
\hline 51 & $(48-60)$ \\
\hline 55 & $(50-63)$ \\
\hline 82 & $(79-90)$ \\
\hline
\end{tabular}

Figura 2. A: tasas de remisión clínica bajo el tratamiento de vedolizumab en la semana 14 y B: el mes 12 en los pacientes con colitis ulcerosa y enfermedad de Crohn sin tratamiento previo de anti-factor de necrosis tumoral alfa/ corticosteroides.

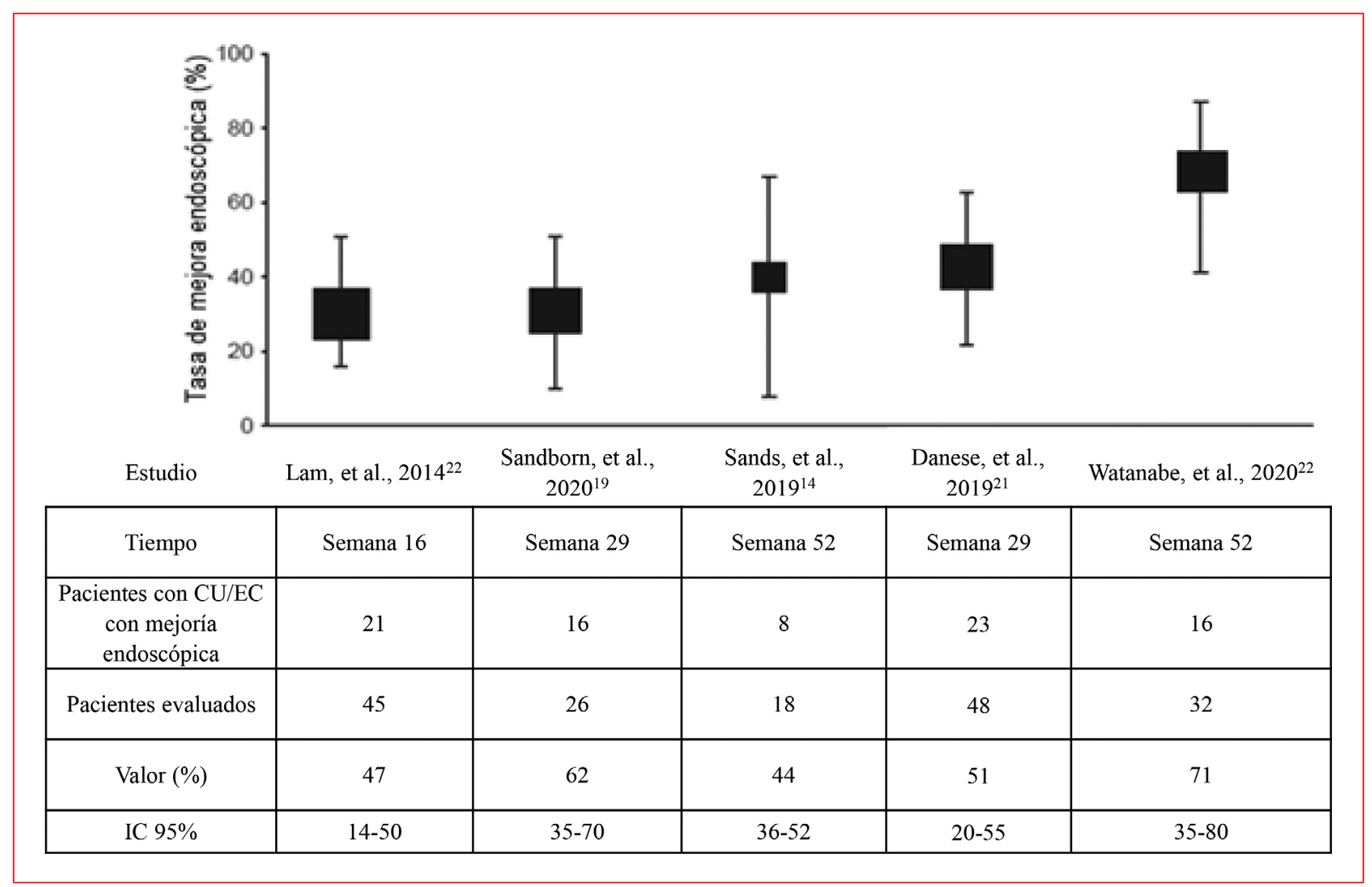

Figura 3. Tasas de la mejora endoscópica del paciente con colitis ulcerosa (CU) y enfermedad de Crohn (EC) bajo el tratamiento de vedolizumab. 
Tabla 3. Seguridad del tratamiento con vedolizumab en los diferentes estudios

\begin{tabular}{|c|c|c|c|}
\hline Evento adverso (EA) & $\begin{array}{l}\text { Pacientes con EA a } \\
\text { los } 6 \text { meses }(\%)\end{array}$ & $\begin{array}{l}\text { Pacientes con EA a } \\
\text { los } 12 \text { meses }(\%)\end{array}$ & Estudios \\
\hline Artralgia & 10 & 15 & $\begin{array}{l}\text { Lam, et al., 2014 }{ }^{16} \text {; Sandborn, et al., } 2020^{19} \text {; Sands, } \\
\text { et al., 2019 }\end{array}$ \\
\hline Dolor de cabeza & 8 & 12 & Loftus, et al., 202017; Motoya, et al., $2019^{18}$ \\
\hline Fatiga & 15 & 22 & $\begin{array}{l}\text { Lam, et al., 2014 }{ }^{16} \text {; Sandborn, et al., 202019; Sands, et al., } \\
\text { 201914; Loftus, et al., 202017; Motoya, et al., } 2019^{18}\end{array}$ \\
\hline Exacerbación & 4 & 8 & Lam, et al., $2014^{16}$ \\
\hline Fiebre & 1 & 4 & $\begin{array}{l}\text { Motoya, et al., 201918; Danese, et al., 201921; Sandborn, } \\
\text { et al., 202019 }\end{array}$ \\
\hline Náuseas & 5 & 8 & Loftus, et al., $2020^{17}$ \\
\hline $\begin{array}{l}\text { Infección por Clostridium } \\
\text { difficile }\end{array}$ & 8 & 10 & Motoya, et al., 201918; Sandborn, et al., $2020^{19}$ \\
\hline $\begin{array}{l}\text { Otras infecciones } \\
\text { gastrointestinales }\end{array}$ & 5 & 5 & Sands, et al., 201914; Danese, et al., $2019^{21}$ \\
\hline Infecciones respiratorias & 2 & 4 & Loftus, et al., $2020^{17}$; Watanabe, et al., $2020^{22}$ \\
\hline $\begin{array}{l}\text { Infecciones del tracto } \\
\text { genitourinario }\end{array}$ & 1 & 1 & Loftus, et al., $2020^{17}$ \\
\hline
\end{tabular}

sin tratamiento previo con anti-TNF- $\alpha$. Los análisis post hoc de los datos de GEMINI indicaron mayores tasas de remisión a los 12 meses en pacientes sin tratamiento previo con anti-TNF- $\alpha$ en comparación con los fracasos de la terapia anti-TNF- $\alpha^{25}$. Varios estudios han demostrado mejores resultados con VDZ en pacientes sin tratamiento previo con anti-TNF- $\alpha$ vs. pacientes tratados con anti-TNF- $\alpha^{26-30}$. Los resultados del estudio actual son consistentes con estos hallazgos.

Por otro lado, la curación de la mucosa es un objetivo importante en el tratamiento de la Ell asociado con una remisión clínica sostenida ${ }^{31-33}$. Las recientes directrices clínicas de tratamiento establecen que solo los pacientes con cicatrización de la mucosa, es decir, ausencia de signos macroscópicos de inflamación activa, sin signos o síntomas muy leves o sin signos y síntomas deben considerarse remitidos ${ }^{33}$. Seis de los estudios seleccionados indican que más de la mitad de los pacientes con Ell lograron la curación de la mucosa a los 12 meses $^{34}$. Aunque los datos se limitaron a seis estudios, las tasas observadas de curación de la mucosa durante 12 meses fueron mayores que las tasas combinadas de remisión clínica, lo que respalda los informes anteriores de una falta de correlación clara entre las medidas de los síntomas clínicos y el daño intestinal evaluado por endoscopia ${ }^{35-37}$.

Adicionalmente, en estudios previos se ha demostrado la eficacia y seguridad del VDZ en la CU y la EC; los resultados obtenidos en el presente análisis corroboran que el uso de VDZ tiene un perfil de seguridad favorable en los pacientes con Ell. La artralgia y la fatiga estuvieron entre los eventos adversos más comunes. Este perfil de tolerabilidad puede ayudar a mejorar la persistencia del tratamiento ${ }^{38-41}$, afectando así potencialmente de forma positiva los resultados a largo plazo, aunque la infección por $C$. difficile y el dolor de cabeza también deben tenerse en cuenta al momento de la medicación, así como el monitoreo de la respuesta, una farmacovigilancia continua.

\section{Conclusiones}

Los resultados obtenidos de este metaanálisis confirman la eficacia del VDZ para inducir la respuesta clínica a largo plazo, la remisión clínica sin corticosteroides o sin uso previo con anti-TNF- $\alpha$ y la curación de la mucosa en pacientes con CU y la EC activa de moderada a grave. Los datos de seguridad presentados aquí respaldan el perfil beneficioso a largo plazo del VDZ en el tratamiento de la CU y la EC.

\section{Financiamiento}

La presente investigación no ha recibido ninguna beca específica de agencias de los sectores público, comercial o sin ánimo de lucro. 


\section{Conflicto de intereses}

No existen conflictos de intereses para la presentación de este manuscrito.

\section{Responsabilidades éticas}

Protección de personas y animales. Los autores declaran que para esta investigación no se han realizado experimentos en seres humanos ni en animales.

Confidencialidad de los datos. Los autores declaran que han seguido los protocolos de su centro de trabajo sobre la publicación de datos de pacientes.

Derecho a la privacidad y consentimiento informado. Los autores declaran que en este artículo no aparecen datos de pacientes.

\section{Bibliografía}

1. Ordás I, Eckmann L, Talamini M, Baumgart DC, Sandborn WJ. Ulcerative colitis. Lancet. 2012; 380(9853):1606-19.

2. Da Silva BC, Lyra AC, Rocha R, Santana GO. Epidemiology, demographic characteristics and prognostic predictors of ulcerative colitis. World J Gastroenterol. 2014;20(28):9458-67.

3. Fudman DI, Sattler L, Feuerstein JD. Inpatient management of acute severe ulcerative colitis. J Hosp Med. 2019;14(12):766-73.

4. Elkjaer M, Shuhaibar M, Burisch J, Bailey Y, Scherfig H, Laugesen B, et al. E-health empowers patients with ulcerative colitis: a randomised controlled trial of the web-guided 'Constant-care' approach. Gut. 2010;59(12):1652-61.

5. Wehkamp J, Stange EF. Recent advances and emerging therapies in the non-surgical management of ulcerative colitis. F1000Res. 2018;7:F1000 Faculty Rev-1207.

6. Le Berre C, Roda G, Nedeljkovic Protic M, Danese S, Peyrin-Biroulet L. Modern use of 5-aminosalicylic acid compounds for ulcerative colitis. Expert Opin Biol Ther. 2020;20(4):363-78.

7. Salice M, Rizzello F, Calabrese C, Privitera Hrustemovic H, Gionchetti P. Budesonide MMX: efficacy and safety profile in the treatment of ulcerative colitis. Expert Rev Gastroenterol Hepatol. 2019;13(7):607-13.

8. Gajendran M, Loganathan P, Jimenez G, Catinella AP, Ng N, Umapathy C, et al. A comprehensive review and update on ulcerative colitis. Dis Mon. 2019;65(12):100851.

9. Pugliese D, Felice C, Papa A, Gasbarrini A, Rapaccini GL, Guidi L, et al. Anti TNF- $\alpha$ therapy for ulcerative colitis: current status and prospects for the future. Expert Rev Clin Immunol. 2017;13(3):223-33.

10. Furfaro F, Bezzio C, Ardizzone S, Massari A, de Franchis R, Maconi G. Overview of biological therapy in ulcerative colitis: current and future directions. J Gastrointestin Liver Dis. 2015;24(2):203-13.

11. Guo $C$, Wu K, Liang $X$, Liang $Y$, Li R. Infliximab clinically treating ulcerative colitis: A systematic review and meta-analysis. Pharmacol Res. 2019;148:104455

12. Nalagatla N, Falloon K, Tran G, Borren NZ, Avalos D, Luther J, et al Effect of accelerated infliximab induction on short- and long-term outcomes of acute severe ulcerative colitis: A retrospective multicenter study and meta-analysis. Clin Gastroenterol Hepatol. 2019;17(3):502-509.e1.

13. Sandborn WJ, van Assche G, Reinisch W, Colombel JF, D'Haens G, Wolf DC, et al. Adalimumab induces and maintains clinical remission in patients with moderate-to-severe ulcerative colitis. Gastroenterology. 2012;142(2):257-65.e1-3.

14. Sands BE, Sandborn WJ, Panaccione R, O'Brien $C D$, Zhang $H$, Johanns $\mathrm{J}$, et al. Ustekinumab as induction and maintenance therapy for ulcerative colitis. N Engl J Med. 2019;381(13):1201-14.

15. Feagan BG, Rutgeerts $P$, Sands BE, Hanauer S, Colombel JF, Sandborn WJ, et al. Vedolizumab as induction and maintenance therapy for ulcerative colitis. N Engl J Med. 2013;369(8):699-710.

16. Lam MC, Bressler B. Vedolizumab for ulcerative colitis and Crohn's disease: results and implications of GEMINI studies. Immunotherapy. 2014;6(9):963-71.
17. Loftus EV Jr, Sands BE, Colombel JF, Dotan I, Khalid JM, Tudor D, et al. Sustained corticosteroid-free clinical remission during vedolizumab maintenance therapy in patients with ulcerative colitis on stable concomitant corticosteroids during induction therapy: A post hoc analysis of GEMINI 1. Clin Exp Gastroenterol. 2020;13:211-20.

18. Motoya S, Watanabe K, Ogata H, Kanai T, Matsui T, Suzuki Y, et al. Vedolizumab in Japanese patients with ulcerative colitis: A phase 3, randomized, double-blind, placebo-controlled study. PloS One. 2019;14(2):e0212989.

19. Sandborn WJ, Baert F, Danese S, Krznarić Ž, Kobayashi T, Yao X, et al. Efficacy and safety of vedolizumab subcutaneous formulation in a randomized trial of patients with ulcerative colitis. Gastroenterology. 2020;158(3):562-72.e12.

20. Sands BE, Sandborn WJ, Van Assche G, Lukas M, Xu J, James A, et al. Vedolizumab as induction and maintenance therapy for Crohn's disease in patients naïve to or who have failed tumor necrosis factor antagonist therapy. Inflamm Bowel Dis. 2017;23(1):97-106.

21. Danese S, Sandborn WJ, Colombel JF, Vermeire S, Glover SC, Rimola J, et al. Endoscopic, radiologic, and histologic healing with vedolizumab in patients with active Crohn's disease. Gastroenterology. 2019;157(4):1007-18.e7.

22. Watanabe K, Motoya S, Ogata H, Kanai T, Matsui T, Suzuki Y, et al. Effects of vedolizumab in Japanese patients with Crohn's disease: a prospective, multicenter, randomized, placebo-controlled Phase 3 trial with exploratory analyses. J Gastroenterol. 2020;55(3):291-306.

23. Sands BE, Peyrin-Biroulet L, Loftus EV Jr, Danese S, Colombel JF, Törüner M,et al. Vedolizumab versus adalimumab for moderate-to-severe ulcerative colitis. N Engl J Med. 2019;381(13):1215-26.

24. Berends SE, Strik AS, Löwenberg M, D’Haens GR, Mathôt RAA. Clinical pharmacokinetic and pharmacodynamic considerations in the treatment of ulcerative colitis. Clin Pharmacokinet. 2019:58(1):15-37.

25. Favale A, Onali S, Caprioli F, Pugliese D, Armuzzi A, Macaluso FS, et al. Comparative efficacy of vedolizumab and adalimumab in ulcerative colitis patients previously treated with infliximab. Inflamm Bowel Dis. 2019;25(11):1805-12.

26. Feagan BG, Schreiber S, Wolf DC, Axler JL, Kaviya A, James A, et al. Sustained clinical remission with vedolizumab in patients with moderate-to-severe ulcerative colitis. Inflamm Bowel Dis. 2019 May 4;25(6):1028-35.

27. Scribano ML. Vedolizumab for inflammatory bowel disease: From randomized controlled trials to real-life evidence. World J Gastroenterol. 2018;24(23):2457-67.

28. Loftus EV Jr, Colombel JF, Feagan BG, Vermeire S, Sandborn WJ, Sands BE, et al. Long-term efficacy of vedolizumab for ulcerative colitis. J Crohns Colitis. 2017;11(4):400-11.

29. Novak G, Hindryckx P, Khanna R, Jairath V, Feagan BG. The safety of vedolizumab for the treatment of ulcerative colitis. Expert Opin Drug Saf. 2017;16(4):501-7.

30. Takatsu N, Hisabe T, Higashi D, Ueki T, Matsui T. Vedolizumab in the treatment of ulcerative colitis: An evidence-based review of safety, efficacy, and place of therapy. Core Evid. 2020;15:7-20.

31. Lukin D, Faleck D, Xu R, Zhang Y, Weiss A, Aniwan S, et al. Comparative safety and effectiveness of vedolizumab to tumor necrosis factor antagonist therapy for ulcerative colitis. Clin Gastroenterol Hepatol. 2020:S1542-3565(20)31388-4.

32. Boal Carvalho P, Cotter J. Mucosal healing in ulcerative colitis: A comprehensive review. Drugs. 2017;77(2):159-73.

33. Shahidi N, Bressler B, Panaccione R. Vedolizumab for the treatment of ulcerative colitis. Expert Opin Biol Ther. 2016;16(1):129-35.

34. Battat R, Ma C, Jairath V, Khanna R, Feagan BG. Benefit-risk assessment of vedolizumab in the treatment of Crohn's disease and ulcerative colitis. Drug Saf. 2019;42(5):617-32.

35. McLean LP, Cross RK. Pharmacodynamic assessment of vedolizumab for the treatment of ulcerative colitis. Expert Opin Drug Metab Toxicol. 2016;12(7):833-42.

36. Hernandez L, Kuwabara H, Shah A, Yamabe K, Burnett H, Fahrbach K, et al. Cost-effectiveness analysis of vedolizumab compared with other biologics in anti-TNF-naïve patients with moderate-to-severe ulcerative colitis in Japan. Pharmacoeconomics. 2020;38(1):69-84.

37. Sands BE, Feagan BG, Rutgeerts P, Colombel JF, Sandborn WJ, Sy R, et al. Effects of vedolizumab induction therapy for patients with Crohn's disease in whom tumor necrosis factor antagonist treatment failed. Gastroenterology. 2014:147(3):618-27.e3.

38. Vermeire S, Lukáš M, Magro F, Adsul S, Lindner D, Rosario M, et al. Vedolizumab efficacy, safety, and pharmacokinetics with reduced frequency of dosing from every 4 weeks to every 8 weeks in patients with Crohn's disease or ulcerative colitis. J Crohns Colitis. 2020;14(8):1066-73.

39. Dart RJ, Samaan MA, Powel N, Irving PM. Vedolizumab: toward a personalized therapy paradigm for people with ulcerative colitis. Clin Exp Gastroenterol. 2017;10:57-66.

40. O'Connor DB, Lahiff C. Vedolizumab versus adalimumab for moderate-to-severe ulcerative colitis. N Engl J Med. 2020;382(1):92-3.

41. Rietdijk ST, D'Haens GR. Vedolizumab for the treatment of ulcerative colitis. Expert Rev Clin Pharmacol. 2014;7(4):423-30. 